\title{
Energy efficient outdoor lighting: an implementation
}

\author{
Subtitle as needed (paper subtitle)
}

\author{
Ahmed Farahat, Anna Florea, Jose L. Martinez \\ Lastra \\ Tampere University of Technology \\ Tampere, Finland \\ jose.lastra@tut.fi
}

\author{
Christian Brañas Reyes, Francisco J. Azcondo \\ University of Cantabria \\ Santander, Spain \\ branasc@unican.es
}

\begin{abstract}
Lighting strongly influences human daily activities. Smart lighting emerged as new generation of lighting system, able to precisely address the specific needs. Under conditions of shortage in fossil fuels and high prices for energy, smart lighting enables design of lighting systems, which can efficiently use power resources without compromising the user experience.

The paper describes implementation of outdoor lighting solution targeting energy efficiency. The proposed approach takes holistic perspective on efficiency and considers light source selection and power management along with control scenarios for lighting scene rendering. System architecture is designed following the Internet of Things vision.
\end{abstract}

Keywords-Smart lighting; energy efficiency; Key Performance Indicators; smart metering; interoperability

\section{INTRODUCTION}

Due to the non-renewable energy related impacts on economy and environment, the urge for attaining energy efficient applications is becoming a critical issue. The lighting sector alone consumes around $25 \%$ of the total energy in commercial buildings in the US and, $19 \%$ of the global energy consumption. This is considered to be more than energy produced by hydro or nuclear power plants and, also equivalent to energy produced from natural gas [1]. Therefore drastic measures should be taken, in order to reduce lighting energy consumption for preserving energy resources and reduce environmental impact.

Smart lighting is one of the solutions for achieving adaptability of lights according to user requirements while reducing the energy consumption at the same time. Smart lighting systems involve complex networks of wireless sensors, dimmers and meters operated by advanced control applications for achieving efficiency goals. These systems are considered the next generation of standard lighting systems, as it will provide better performance, efficiency and sustainability. [2]

The development of smart lighting is conducted on two phases [3]: "First wave" and "Second wave". The "First wave" stands for the replacement of standard bulbs with the new LED technology. The progress in this wave is progressing in the form of researching new technologies in order to reach a better efficiency, cost reduction, prolonged lifetime and chip design of the LED technology. The "Second wave" of development is concerned with the controllability, fast response and control communications of the LED, which will lead to revolutionizing the adaptive lighting to reach higher efficiency and satisfy user comfort.

This paper presents a use case for implementing and monitoring an outdoor efficient smart lighting. The paper discusses all aspects of efficiency for the implementation and possibilities for improving it in the future. In the next section a brief summary for lighting efficiency. Then in section III the use case is described from the power management, control and monitoring point of views. Section IV explains the experiments conducted and discusses the results. Finally section $\mathrm{V}$ gives a summary of the paper and discusses future improvements.

\section{EFFICIENCY IN OUTDOOR LIGHTING}

Energy efficiency is defined as measurement of the performance of a device or system and, it is defined as the ratio of the output to input energies [4]. Energy efficiency of the outdoor lighting systems is influenced by such factors as: efficiency of light sources, the driver used to supply and control the light source, power factor, effect of weather conditions, and awareness of the user needs for specific lighting conditions [5].

\section{A. Light Sources}

In the early years lighting was limited to incandescent and fluorescent lamps until, N. Holonyak successfully generated the first visible red light from a p-n junction as known right now by Light Emitting Diode (LED) [6]. LED lamps have shown rapid developments in the recent years, such that they are integrated in everyday applications. Now LED light sources are generating more light, become more efficient and, produce white light of various colour temperatures [7]. Colour rendering is another key factor to define the light source performance consistent to the perception of the human eye. Another lighting source produced from organic substances, Organic Light Emitting Diodes (OLED) gains more attention due to the lighting quality characteristics and physical characteristics of the light source [8]. However, OLED is still a newly born technology thus, it neither reached the efficiency nor lifetime of LEDs, which have been developing for more than 50 years. 


\section{B. Lighting requirments}

Need for particular lighting scene (i.e. amount, direction, and quality of light) varies dramatically depending on the activities performed. Awareness of the user needs allows adjusting the performance of lighting application, hence creating another opportunity for energy savings.

One of the main factors affecting the visibility is illuminance. The illuminance is the amount of light flux falling on a certain area, and it is measured in in lux or foot candle (fc). The required illuminance varies depending on the task performed, reflectance in the area and age of worker. The American National Standards Institute (ANSI) defined a set of illumination ranges required based on the performed task [9].

\section{Lighting scenes and control scenarios}

Lighting requirements are met with help of control applications. Simple light control applications rely on manual control and control algorithms that follow a pre-programmed schedule. Smart lighting applications adjust the lighting scenes based on values of the monitored parameters. There are several methods used to obtain the information about the lit area in order to infer the required light conditions including motion detection, light detection, and vision. The most common method is the light detection, while the most expensive is vision.

\section{IMPLEMENTATION}

The proposed smart lighting solution utilizes a set of dimmable lamps, energy analysers, RTU controller, sensors and a Raspberry Pi unit, in order to achieve the desired goals, as illustrated in Figure 1 Solution overview.

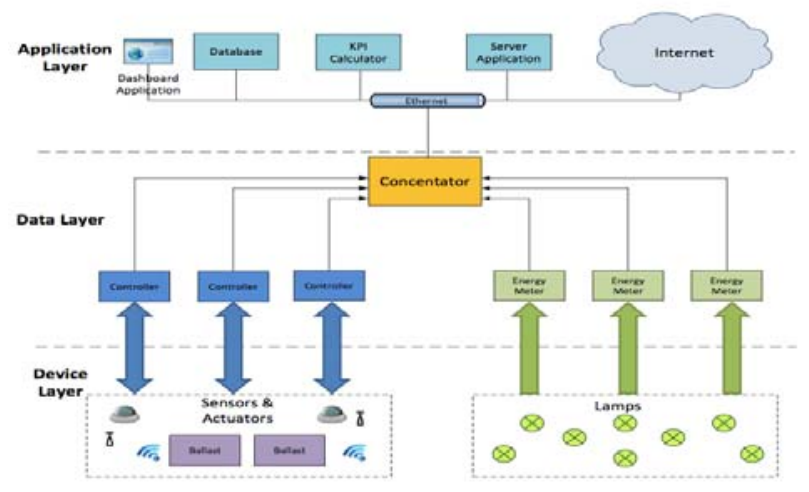

Fig. 1. Solution overview

\section{A. Power management}

The proposed driver is a two stages solution including a power factor correction (PFC), and a high dynamic performance resonant converter. The PFC rectifies the utility voltage with nearly unity power factor and supplying the second stage with a DC bus voltage $\left(V_{\text {bus }}\right)$. A two-phase resonant converter circuit operating with phase shift modulation at constant switching frequency, as shown in Fig. 2 , is adopted for this application. The fast response of the converter that supply the LED string current simplifies the control action to compensate for the $V_{\text {bus }}$ ripple as well as to implement a pulse-width modulated (PWM) dimming. The design accepts a quite large $V_{b u s}$ ripple to reduce the DC voltage capacitance and avoid electrolytic capacitors due to their shorter lifetime. DC bus voltage ripple is compensated by means of a feed forward action while the LED current amplitude is set by a feedback controller. The PWM dimming frequency is selected above the visible and non-visible perception of the human eye and the pulse width is defined according to the commands received from the internet based interface. Since the LED current amplitude controlled to be constant, the LED colour rendering is preserved, while producing energy savings.

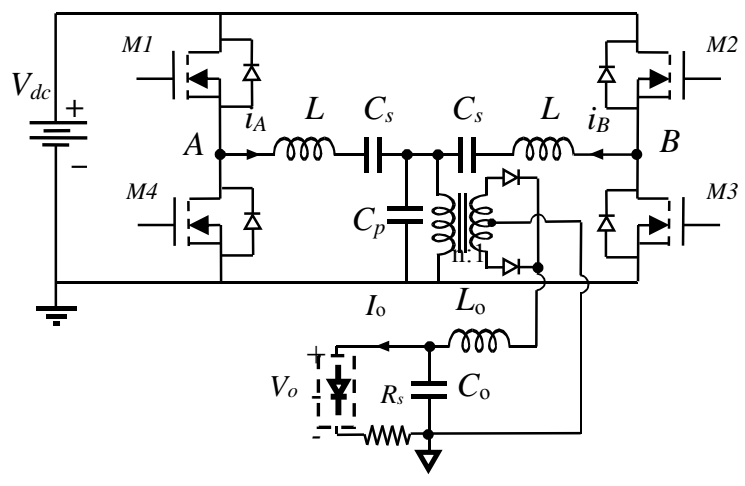

Fig. 2. Two-phase LCC resonant converter with LED string load and resistive current sensor

The voltage at the midpoints $\mathrm{A}$ and $\mathrm{B}$, i.e. $v_{A}$ and $v_{B}$ are $50 \%$ duty cycle square waves impressed by the complementary switching of the transistors $M x$. The LED string current amplitude, $I_{o}$, according to the LED specifications is set by the phase displacement, $\Psi$, between $v_{A}$ and $v_{B}$, as presented in Fig. 3 , with a $10 \mathrm{kHz}$ bandwidth control loop.

$$
I_{o}=\frac{n V_{d c} \sqrt{1+C_{p} / 2 C_{s}}}{Z_{p}} \operatorname{Cos}(\Psi / 2),
$$

with

$$
Z_{p}=\omega_{p} L=\frac{2}{\omega_{p} C_{p}}
$$

and

$$
\omega_{p}=\frac{1}{\sqrt{L C_{p} / 2}}
$$




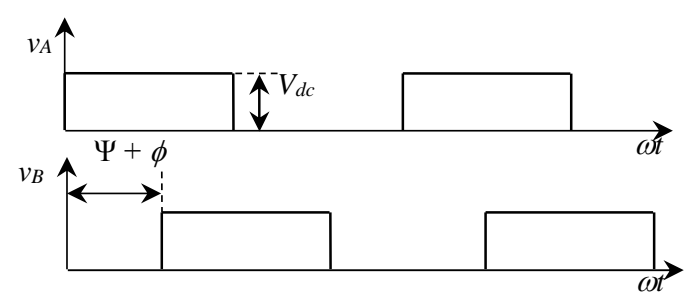

Fig. 3. Midpoint voltages in each inverter leg.

Alternative switching of $\Psi$ between the control command and $180^{\circ}$ provides the PWM dimming requested by the control implemented by the outer layer of the system aimed at combining the right performance at minimum energy consumption.

\section{B. Control}

\section{1) Hardware}

The smart lighting implementation is consisting of 6 fixtures of LED lamps, 1 controller, 2 Energy meters, 1 Valopaa master unit, 1 THL sensor, 1 personnel counter, 1 light and temperature sensor, 1 motion sensors and 1 push button.

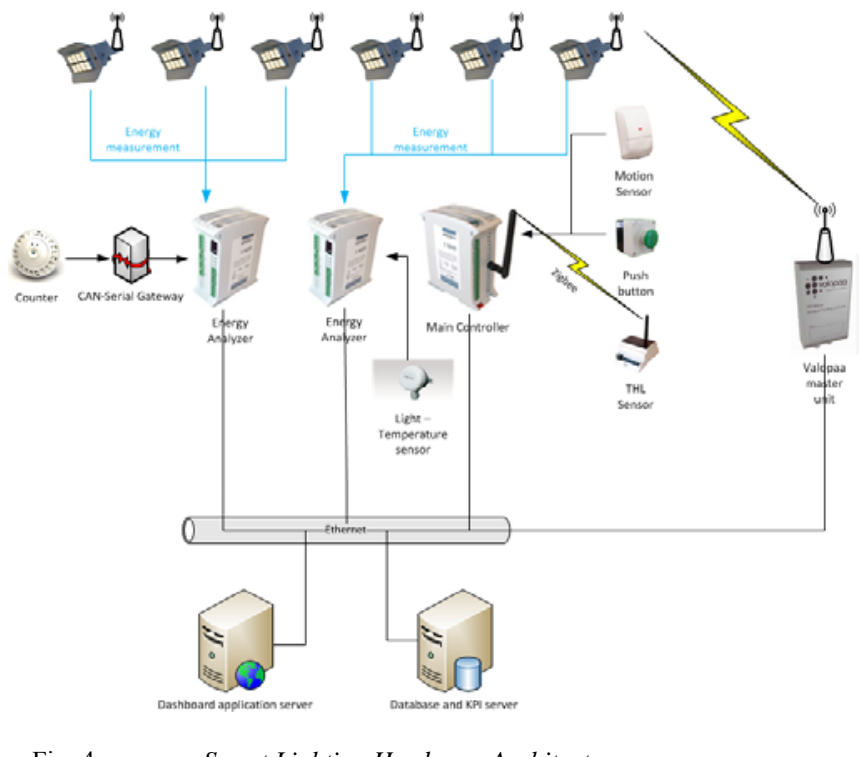

Fig. 4. Smart Lighting Hardware Architecture

\section{a) Lamps and controller:}

The lamps fixtures are of type Valopaa VP3411 LED floodlight which provides adequate amount of luminance with minimum energy dissipation. The lighting fixture can be installed outdoor as it is sealed and durable for use in extreme weather conditions. The aluminium casing provides efficient heat dissipation for the electronics inside. [10] The lamp divers are controlled wirelessly by radio signals by the Valopaa Master Unit controller.
The Master Unit can also generate schedules and give statistical feedback for energy consumptions and lamps temperature through Ethernet. The Master Unit was modified to handle specifically tailored commands for this implementation. This modification will allow control of the lamps via Https POST requests.

\section{b) Controller and Energy meters:}

Control and energy measurement are conducted using S1000 devices, which are smart programmable RTUs with Ethernet connectivity. They can publish web-service events to subscribers in order to monitor system status and consumption. Two of these devices will be extended with E-10 energy module, which supplements energy measurement capabilities for 3 different devices. The controller will be extended with wireless router module, in order to communicate with the THL sensor node. These devices also have analogue and digitals I/O for connecting to the outer world.

\section{c) Sensors:}

A set of sensors are used to sense the current surroundings. A THL sensor is used for sensing the temperature, humidity and ambient light and, sending these values wirelessly using ZigBee technology. Busch-Watchdog 280 MasterLINE is a passive infrared detector used for motion detection of thermal objects. The motion sensor has a range of $18 \mathrm{~m}$ and angle of $280^{\circ}$ to cover all of the backyard area. A visual counter is installed above the entrance door to count personnel going in and out of the facility using thermal image processing. An external light and temperature sensor is installed to get real time values for the outside conditions.

\section{2) Software algorithm}

In order to reduce the energy consumed by the lamps a control algorithm was proposed, which takes into consideration surrounding and industrial parameters, as well as standards and regulation for required illumination levels. The main goal of the control is to deliver the minimum sufficient amount of luminance required by the user without disturbing visibility.

The algorithm considers 3 main variables: weather condition, presence/task performed and, ambient light intensity. The system acquires surrounding weather data from an external service offered by Yahoo!. An infrared passive thermal motion sensor is used to detect presence in the backyard area. An additional pushbutton is installed near the door to trigger the loading/unloading modes, indicating a task is being performed in the area. An indoor light sensor is installed gathers outside ambient light intensity and, publishes the data as a service.

The combinations of those three variables lead to 32 different profile. A profile (Pxyz) is an indication of the system current state where, $\mathrm{X}, \mathrm{Y}$ and $\mathrm{Z}$ represents a numeric values assigned to the aforementioned variables respectively. A lighting scene is assigned to each profile according to the needs and performed task. The lighting scenes were designed according to ANSI standard and the light distribution map of the yard. 
The control is performed by sending a message defining the target lighting levels from the controller device to the application server. Then the server transfers these levels to the lamps controller. This intermediate step has been taken due to the reason that currently available firmware of the RTUs does not support communication over HTTPS.

TABLE I. PROFILES AND VARIABLES ADAPTED FROM [11]

\begin{tabular}{|c|c|c|c|c|c|}
\cline { 2 - 6 } \multicolumn{2}{c|}{} & \multicolumn{4}{c|}{ Weather Condition } \\
\hline Light level & Presence & Clear & Mist & Rain & Snow \\
\hline >Threshold & Personnel & $P_{111}$ & $P_{211}$ & $P_{311}$ & $P_{411}$ \\
\cline { 2 - 6 } & Truck & $P_{121}$ & $P_{221}$ & $P_{321}$ & $P_{421}$ \\
\cline { 2 - 6 } & Both & $P_{131}$ & $P_{231}$ & $P_{331}$ & $P_{431}$ \\
\cline { 2 - 6 } & None & $P_{141}$ & $P_{241}$ & $P_{341}$ & $P_{441}$ \\
\hline$<$ Threshold & \multicolumn{5}{|c|}{$\ldots$} \\
\hline
\end{tabular}

\section{Monitoring}

In order to monitor the performance in real time and analyse the system results, a web based dashboard application was developed. The application offers stability, security, reliability, and user friendliness. There are different interfaces designed specifically according to different user rolls: admin, manager, operator and researcher. The application has many data representation methods such as widgets, gauges and charts, in order to easily interpret the data.

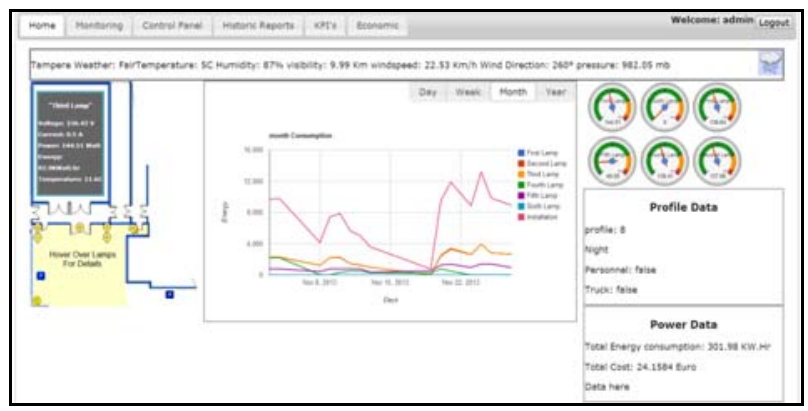

Fig. 5. Monitoring Dashboard

The application was developed in Java environment using Spring, JMEDS and Camel frameworks. Spring framework is used for dependency injection, security and developing the MVC. JMEDS library is used for creating DPWS client for communicating with the devices. Camel framework is used for creating endpoints for receiving the messages and routing them to the desired parser.

The application is deployed on a Tomcat server residing on a Raspberry Pi (RPi) compact computer. The main features for using RPi are: compact size so it can fit in the control cabinet, low power consumption and, adequate computational power for hosting the application server.

\section{PERFORMANCE ASSESSMENT}

Tests were conducted in between $15^{\text {th }}$ of November and $10^{\text {th }}$ of December 2013. During the testing period the average day length in Finland was six hours and the weather was almost clear. The goal of the tests is to compare results between using outdated scheduled system used and applying the smart lighting implementation for adaptive automatic control.

The scheduled lighting control was designed as a reference benchmark for comparing energy consumption as, there were no past energy bills or readings that can be used for previous installation. According to the schedule the lamps should work with $90 \%$ lighting capacity from sunset until midnight then, the levels dim down to $30 \%$ then they turn off completely by sunrise. This schedule showed a constant consumption of $12.4 \mathrm{~kW} . \mathrm{hr}$.

The smart lighting automatic control was applied to the lights starting November $29^{\text {th }}$. Daily energy consumption (SL004) is plotted in Figure 4, where the vertical and horizontal axes represent the energy consumed in Watt.hr and days respectively. It is clear from the plot that in the beginning the consumption was around $12 \mathrm{~kW}$.hr. The lighting levels were refined and waiting time of the sensors was reduced to $10 \mathrm{~s}$, consequently starting December $4^{\text {th }}$ the energy consumption was reduced significantly to $8.4 \mathrm{~kW}$.hr. A problem in the KPI calculation was noticed during December $5^{\text {th }}$ and $6^{\text {th }}$, thus records are ignored for these two days.

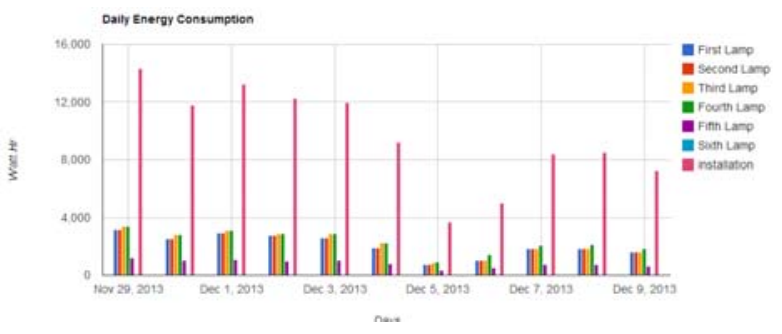

Fig. 6. Daily energy consumption for using automatic control

A comparison between performance of scheduled and adaptive control are presented in Table 1. The adaptive system showed enhanced results in comparison to scheduled one, with efficiency of $33.62 \%$. The energy efficiency is calculated by using equation 1, where "E" stands for energy. Similar equation was applied to the rest of parameters. More tests under different conditions will be conducted in the future work.

$$
\eta_{\%}=\frac{E_{\text {scheduled }}-E_{\text {controlled }}}{E_{\text {scheduled }}} \times 100
$$

TABLE II. SCHEDULED AND CONTROLLER COMPARIOSN

\begin{tabular}{|c|c|c|c|}
\hline $\begin{array}{c}\text { Criteria } \\
\text { per day }\end{array}$ & Scheduled & Controlled & Efficiency \\
\hline Energy & $12.655 \mathrm{~kW} \cdot \mathrm{hr}$ & $8.4 \mathrm{~kW} . \mathrm{hr}$ & $33.62 \%$ \\
\hline $\begin{array}{c}\text { Average } \\
\text { Power }\end{array}$ & $104.845 \mathrm{~W}$ & $84.678 \mathrm{~W}$ & $12.24 \%$ \\
\hline LPD & $0.68 \mathrm{~W} / \mathrm{m}^{2}$ & $0.553 \mathrm{~W} / \mathrm{m}^{2}$ & $12.24 \%$ \\
\hline $\begin{array}{c}\mathbf{C O}_{2} \\
\text { Equivalent }\end{array}$ & $8.929 \mathrm{Kg}$ & $5.921 \mathrm{Kg}$ & $33.62 \%$ \\
\hline Cost & $1.012 €$ & $0.672 €$ & $33.62 \%$ \\
\hline
\end{tabular}




\section{CONCLUSION}

This paper presented a smart lighting implementation for achieving better overall efficiency. A control algorithm was developed with the intention of reducing the energy consumption and, taking into account all surrounding variables to achieve adaptability of the lights and improve user experience. The light sources were selected according to their performance, effective quantity of light, heat dissipation and, encapsulation to withstand difficult weather conditions. The LED driver has been tested and has shown an outstanding robustness and performance for power management and reducing consumption while preserving the light quality. The system performance was measured and analysed in the web application and, the amount of energy savings and cost reduction was demonstrated.

\section{ACKNOWLEDGMENT}

This work is co-sponsored by the Spanish Ministry of Science and the EU through the project CICYT-FEDER- TEC2011-

23612: "Power conversion with new digital control techniques and soft-saturation magnetic cores"

\section{REFERENCES}

[1] Aalto University School of Science and Technology, Guidebook on Energy Efficient Electric Lighting for Buildings, Raisio: IEA publications, 2010
[2] M. Miki, T. Hiroyasu and K. Imazato, "Proposal for an intelligent lighting system, and verification of control method effectiveness," Cybernetics and Intelligent Systems, vol. 1, pp. 520-525, 2004.

[3] R. Karlicek, "Smart lighting - more than illumination," in Asia Communications and Photonics Conference (ACP), Guangzhou, 2012.

[4] M. Burgos-Payan, F. Correa-Moreno and J. Riquelme-Santos, "Improving the energy efficiency of street lighting. A case in the South of Spain," in 9th International Conference on theEuropean Energy Market (EEM), Florence, 2012

[5] D. Maheswaran, K. Kailas, V. Rangaraj and W. Kumar, "Energy efficiency in electrical systems," in IEEE International Conference on Power Electronics, Drives and Energy Systems (PEDES), Bengaluru, 2012.

[6] N. Holonyak and S. F. Bevacqua, "COHERENT (VISIBLE) LIGHT EMISSION FROM Ga(As1-xPx) JUNCTIONS,” Appl. Phys. Lett., vol. 1, no. 4, pp. 82-83, 1962.

[7] M. Shur and A. Zukauskas, "Solid-State Lighting: Toward Superior Illumination," Proceedings of the IEEE, vol. 93, no. 10, pp. 1691-1703, 2005 .

[8] S. Kunić and Z. Šego, "OLED technology and displays," in ELMAR, Zadar, 2012

[9] J. Anshel, Visual Ergonomics Handbook, Boca Raton: CRC Press Taylor and Francis Group, 2005.

[10] Valopaa Energy Efficient Lighting, "VP3411 LED floodlight fixture," [Online]. Available: http://www.valopaa.com/led_lighting_products/intelligent_led_lighting system/vp3411i_led_floodlight. [Accessed 21 September 2013].

[11] A. Florea, A. Farahat, C. Postelnicu, J. L. M. Lastra and F. J. A. Sánchez, "Smart Lighting in Multipurpose Outdoor Environments: Energy Efficient Solution using Network of Cooperating Objects.," in 4th International Workshop on Networks of Cooperating Objects for Smart Cities 2013 (CONET/UBICITEC), Philadelphia, 2013. 